\title{
Vinyl Acetate/butyl acrylate/acrylate Research of Ternary Soap-free Emulsion Polymerization
}

\author{
Li-guang XIAO ${ }^{1, a}$ and Meng $\mathrm{ZHANG}^{2, \mathrm{~b}}$ \\ ${ }^{1,2}$ College of Material Science and Engineering, Jilin Jianzhu University, Changchun,China \\ axlg627@163.com , ${ }^{\mathrm{b}} 1369171924 @ q q . c o m$
}

\begin{abstract}
Through the vinyl acetate/butyl acrylate/acrylic acrylic emulsion preparation without soap vinegar, with solid content, gel, emulsion stability and film forming properties and tensile strength as the main index to study the effect of raw materials on the properties of emulsion. Through the infrared spectrometer soap-free emulsion for microscopic analysis research. Study of the ternary soap-free vinegar acrylic emulsion with good performance.
\end{abstract}

Keywords: soap-free emulsion polymerization; reaction mechanism.

\section{Introduction}

Traditional way of emulsion polymerization as a result of the existence of emulsifier and compactness of film adhesion, water resistance, and gloss and so on all has certain influence, In comparison, soap-free emulsion polymerization it is possible to prepare the surface clean, highly mono-disperse polymer latex particles. Vinegar acrylic emulsion adhesives belong to a class of very broad application of emulsion-type adhesive, which has a high transparency and excellent anti-aging properties, However, vinyl acetate and butyl acrylate emulsion polymerization of monomers binary, because reactivity ratio difference between the larger of the two monomers, The resulting soap-free vinegar $\mathrm{C}$ emulsion stability and water resistance is very poor; Therefore, this experiment was added to this functional acrylic monomer[1], the use of anion - non-ionic reactive ternary system copolymer emulsion polymerization[2] to prepare a good adhesion, stability and other aspects of performance Non-soap vinegar C emulsion.

\section{Experimental Procedures}

\subsection{Raw Material Specifications}

Vinyl acetate; Butyl acrylate; Acrylate; Ammonium persulfate; Methacryl sulfonate; Polyoxyethylene allyl methyl ether; $\mathrm{NaOH}$. 


\subsection{Laboratory Apparatus}

Japan's Shimadzu IRAffinity-1 type Fourier transform infrared spectrometer; JL-1155 laser particle size distribution tester of Research and design institute of light industry in Sichuan province of China.

\subsection{Emulsion Polymerization Method}

To four with a stirrer inside the flask adding sodium methyl acrylic acid and methyl allyl polyoxyethylene ether, put the flask in a water bath pot heating and began to stir. When the temperature is maintained at the set temperature, the first trickle 1/6 monomer, half an hour after dropping $1 / 2$ of ammonium persulfate initiator, and then slowly added dropwise remaining portion of the monomer and initiator. Later titration is complete, the temperature was raised $4.5^{\circ} \mathrm{C}$, after two hours the thermostat to cool down to about room temperature, adjust the $\mathrm{pH}$ with emulsion $1 \mathrm{~mol} / \mathrm{L} \mathrm{NaOH}$ solution at about $7 \sim 8$, filtered receipts.

\section{Results and Discussion}

\subsection{The influence of the functional monomer acrylic acid for soap-free emulsion performance}

By increasing the amount of functional acrylic monomer, we observe trends soap-free emulsion gel rate and conversion rates, and average particle size(Fig.1, Fig.2).

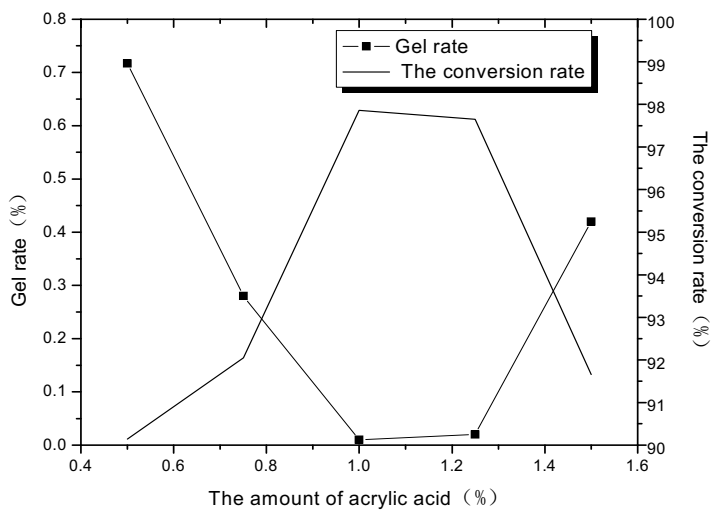

Fig. 1 The amount of acrylic acid on the impact of gel rate and conversion rate of emulsion

Figure 1 shows that, with the increase in the amount of functional acrylic monomer, the resulting free emulsion gel initially decreased after presenting increased the lead after conversion increased and then decreased. This is due to join the acrylic acid as functional monomer, acrylic acid soluble in water and oil, is advantageous to the race of rate of large difference of monomer copolymerization reaction, improve the conversion rate without soap emulsion. According to homogeneous nucleation mechanism[3], the $\mathrm{COOH}$ functional groups of the acrylic acid molecules easily hydrolyzed to the $\mathrm{COO}^{-}$, formed the electrostatic repulsion between latex particles gradually strengthen, improve the stability of the soap-free emulsion. In addition, after the end of the polymerization process, the outer surface of the latex particles surrounded by a large number of carboxyl group, made of 
latex particles and the water molecules, and easy to form hydrogen bonds between latex particles between molecular interactions between the strengthen, improve the film forming properties of the emulsion. However, when the amount of excess acrylic acid, can cause hard monomer soluble in water vinyl acetate macromolecular[4] chain link around the struggling plenty of latex particles, the gel increased. And adding too much acrylic cause low $\mathrm{pH}$ within the system, making vinyl acetate easily hydrolyzed form acetic acid, reducing the conversion rate.

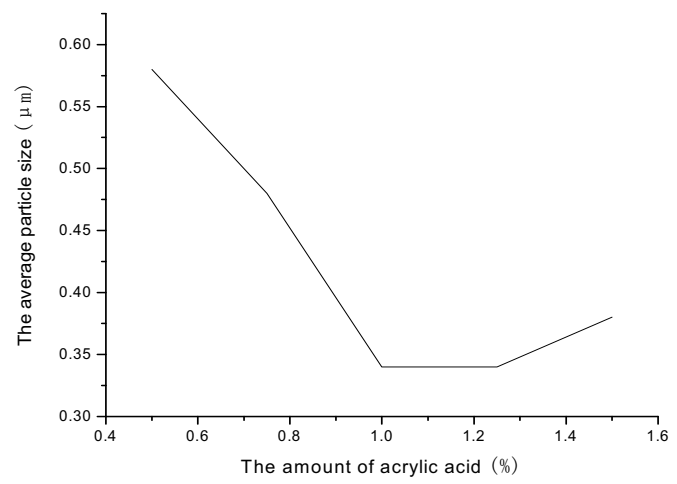

Fig. 2 The amount of acrylic acid on the impact of the average particle size of emulsion

Figure 2 shows that, with the increase in the amount of functional acrylic monomer, the resulting free emulsion of average particle size decreased firstly and then increasing trend, since the acrylate molecule is easily hydrolyzed to $-\mathrm{COO}^{-}$, the electrostatic repulsion between the latex particles enhanced, so that the particle size of the emulsion is reduced; When the amount of excess acrylic acid, soluble in water can cause a hard vinyl acetate monomer molecular chain link around a lot of crazy latex particles, latex particles to come together, the average particle size increased.

The influence of polymerization temperature on the emulsion performance. Research by the method of temperature rising of soap-free emulsion gel rate and the change of solid content, as show in Figure 3 .

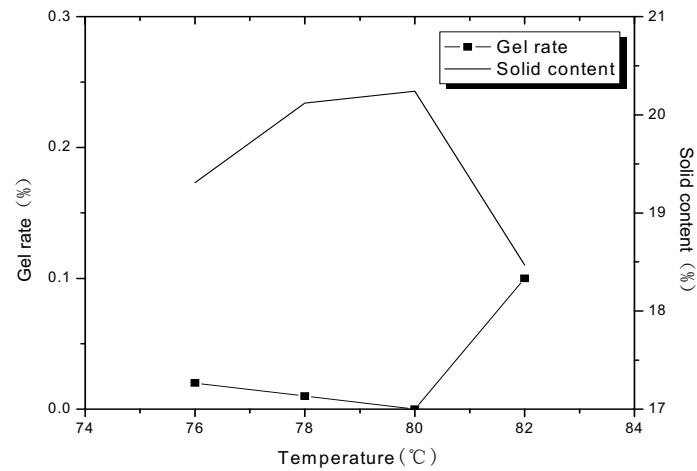

Fig. 3 Effect of polymerization temperature on gel fraction and a solids content of the resulting emulsion 
Figure 3 shows that, as the temperature rises, the resulting free emulsion gel fraction firstly and then decreased and then increased, after solid content first increased and then decreased. This is due to the rise in temperature causes initiator accelerated decomposition of free radicals, a surge in the number of initial particles, so that the polymerization reaction is carried out more perfect; When the temperature rises to a certain degree, the initiator rapid decomposition, forming a large number of chain growth per unit of time, have a dramatic polymerization reaction, a large number of reaction heat is difficult to discharge, automatic acceleration phenomenon occurs--the implosion reaction[5]. And emulsion Brownian motion by particularly large fluctuations, overcome the initial latex particles repulsion and polyether side chain has the space steric effect, reduce the stability of the emulsion and the produce gel.

\subsection{The initiator ammonium persulfate dosage on the properties of the emulsion}

By increasing the dosage of initiator ammonium persulfate, we discuss of soap-free emulsion has the trend of changes in the average particle size and the rate of gel, as show in Figure 4 .

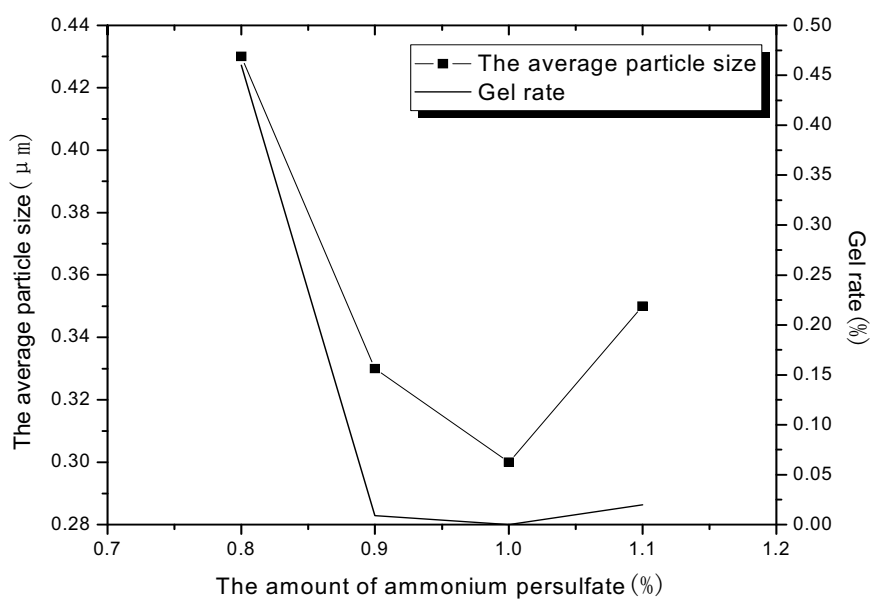

Fig. 4 The amount of ammonium persulfate for the average particle size of emulsion and gel rate impact

The figure 4 shows that with the increase of dosage of initiator ammonium persulfate, the average particle size and gel rate of the resulting emulsion are showing an upward trend after the first reduced. During the polymerization, within a certain range of initiator concentration will gradually increase, accelerates the rate of formation of free radicals, resulting in the polymerization reaction rate is accelerated, a large number of emulsion particles generated. Polymer latex particle nucleation and growth process is divided into initial free radicals, oligomer radical, oligomer micelle, initial latex particles, stable polymer particles, etc. Initiator is increased to a certain extent, it is easy to produce a large number of the initial free radicals, a large number of initial surge latex particles, so that the outer surface of the charge density of the particles decreased, and Zeta potential decrease in 
the polymerization system, resulted in the polymer particle coalescence together, the average particle size increases.

\subsection{No soap vinegar acrylic emulsion through infrared spectrum analysis}

Figure 5 is no soap vinegar acrylic emulsion infrared spectrogram.

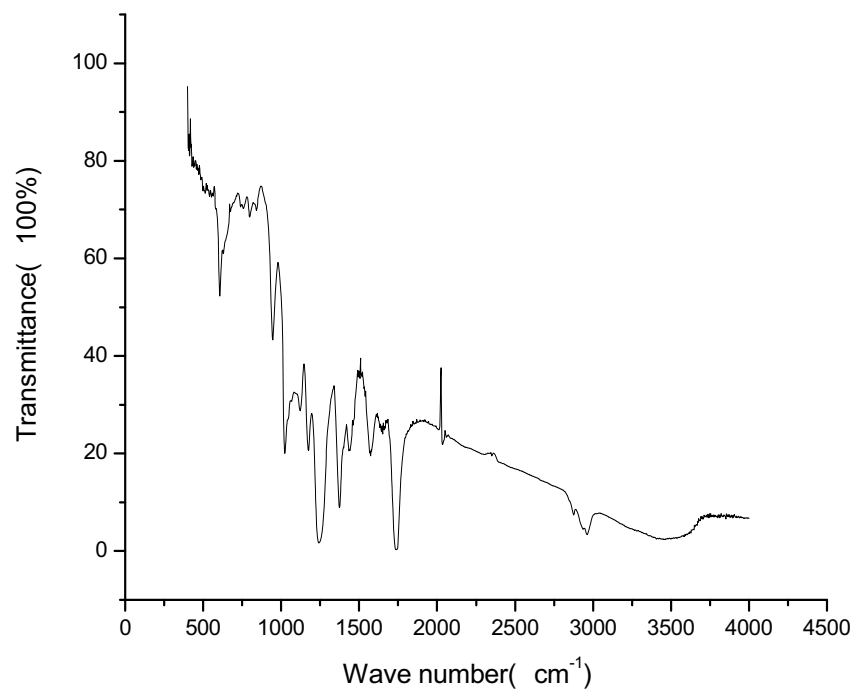

Fig. 5 Soap-free acrylic emulsion vinegar infrared spectrum

Figure 5 shows that, on the spectrum of $802 \mathrm{~cm}^{-1}$ for the multiple $-\mathrm{CH}_{2}$ absorption peak, $1235.0 \mathrm{~cm}^{-1}$ and $1170.7 \mathrm{~cm}^{-1}$ were ester base $(\mathrm{C}-\mathrm{O}-\mathrm{C})$ in the asymmetric stretching vibration absorption peak and symmetric scale absorption peak, $1372.2 \mathrm{~cm}^{-1}, 1438.1 \mathrm{~cm}^{-1}$ methyl and methylene contains $\mathrm{C}-\mathrm{H}$ key in-plane bending vibration absorption peak and surface vibration absorption peak, $1735.1 \mathrm{~cm}^{-1}$ represents an acrylate carbonyl group $(\mathrm{C}=\mathrm{O})$ asymmetric stretching vibration, $2952 \mathrm{~cm}^{-1}$ is the methyl $\mathrm{C}-\mathrm{H}$ bond stretching vibration absorption peak, $3452 \mathrm{~cm}^{-1}$ is $-\mathrm{COOH}$ peaks. From this polymer is derived from vinyl acetate, acrylic acid, butyl acrylate as a monomer and prepared.

\section{Conclusion}

Using vinyl acetate and butyl acrylate and acrylic acid as monomer soap-free emulsion polymerization, non-soap emulsion obtained in all aspects of performance is very good. Research shows that the emulsion adhesive strength, tensile strength, film forming performance and stable performance with the addition of acrylic acid are improved greatly.

\section{Acknowledgement}

This work was financially supported by the Jilin Science and Technology Foundation (No.20140307005SF, No.20150203014SF). 


\section{References}

1. Herrera, Ordonez . J, Olayo R, Methyl methacrylate emulsion polyerization at low monomer concentration: kinetics modeling of nucleation. Particle size distribution and rate of polymerization, J.polym.Sci.Eng. 85 (2001) 76-77.

2. Ying-yu RUI, Wen-ying QI, Xia LU, Cheng-bing YU, Progress in emulsion polymerization system, Acrylate Chemical Technology. 19 (2011) 65-68.

3. Fitch R M, The homogeneous nucleation of polymer colloids, British Polymer Journal. 5 (2007) 467-483.

4. Li-guang XIAO, Meng ZHANG, Progression free soap emulsion polymerization theory and research, Journal of Jilin Jianzhu University. 32 (2015) 5-8.

5. Jun XU, Ma-hong YU, Study on vinyl acetate / maleic acid / butyl acrylate copolymer emulsion, Bonding. 9 (2011) 57-59. 